\section{CANCER EPIGENETICS}

\section{It takes two}

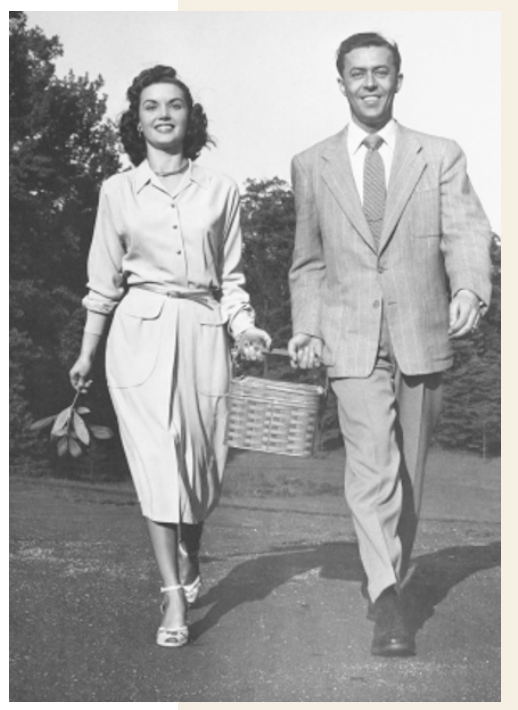

Tumour-suppressor genes act as safeguards that prevent normal cells from forming tumours. But how are they taken out of action during cancer development? Initial findings focused on genetic mutations or deletions. More recent research, however, has unveiled a role for epigenetic events in the inactivation of tumour-suppressor genes. A paper in Nature now sheds further light on the epigenetic mechanisms that operate in human cancer.

An important factor in the control of gene expression is DNA methylation. Several genes that normally suppress tumour development are switched off in human tumours and this is associated with methylation of their promoter regions, but relatively little is known about the mechanisms by which these genes become methylated. The enzymes that catalyse DNA methylation are DNA methyltransferases (DNMTs). The prototype of this enzyme family is Dnmt 1 , which accounts for most DNA methylation in mouse cells. Surprisingly, however, human cells that lack DNMT1 maintain a significant level of overall methylation and gene silencing.

Ina Rhee and colleagues therefore set out to investigate whether other DNMTs might have a more prominent role, and inactivated DNMT3b in a human colorectal carcinoma cell line. As was the case for disruption of DNMT1, the overall levels of genomic DNA methylation dropped by only a smal percentage. By contrast, the simultaneous deletion of DNMT and $D N M T 3 b$ resulted in an almost complete elimination of cellular DNA methyltransferase activity and genomic methylation. Every individual gene locus analysed showed a substantial loss of methylation and increased levels of expression, including several that have been implicated in tumour progression. Furthermore, the proliferation of cells that lack DNMT1 and DNMT3b was markedly reduced. These results clearly establish that methylation is required to shut off tumoursuppressor genes in human cancer cells, and show that the activity of more than one DNA methyltransferase is required to keep tumour suppressors in check if a cell is to escape normal growth control.

\title{
The silent superhero
}

The role of DNA methylation in gene silencing has long been established, but the relationship between DNA methylation and histone methylation was only finally unravelled last year, when it was shown in Neurospora that mutations in a widely conserved $\mathrm{Su}$ (var)-like methyl transferase and in lysine 9 (Lys9) of histone $\mathrm{H} 3$ could abolish all DNA methylation, indicating that DNA methylation is downstream of histone methylation. But is this just a fungus-specific phenomenon? By identifying and characterizing the Arabidopsis $\mathrm{Su}$ (var) homologue, KRYPTONITE (KYP), Jackson et al. now extend the previous findings by showing that this relationship is also conserved in plants.

The study started with a genetic screen for suppressors of a hypermethylated allele of SUPERMAN (SUP), which causes disrupted flower morphology. As well as identifying mutations in CHROMOMETHYLASE3 (CMT3), a known DNA methyltransferase, the screen yielded alleles of another gene, which the authors called KYP. KYP contains a SET domain that is characteristic of $\mathrm{Su}$ (var)9-3 proteins, which are known to be involved in histone
H3 methylation and, as expected, it specifically methylates Lys9 of histone H3. Sequence analysis showed that the activity of the SET domain is either reduced or eliminated in $k y p$ mutants, indicating that this domain is necessary for its function.

Given the dependence of DNA methylation on histone methylation in Neurospora, the authors decided to investigate the effects of loss-of-function mutations in KYP on DNA methylation. They used methylation-sensitive restriction enzymes and bisulphite sequencing to compare the methylation status of several loci, including SUP, which is predominantly methylated at $\mathrm{CpNpG}$ sites, and FLOWERING LOCUS WA, which is mostly methylated at CpG sites. The authors found that, as in the cmt3 mutants, in kyp mutants DNA methylation was affected specifically at $\mathrm{CpNpG}$ sites in all of the sequences tested.

So how is the histone methylation that is brought about by KYP 'translated' into DNA methylation? The fact that

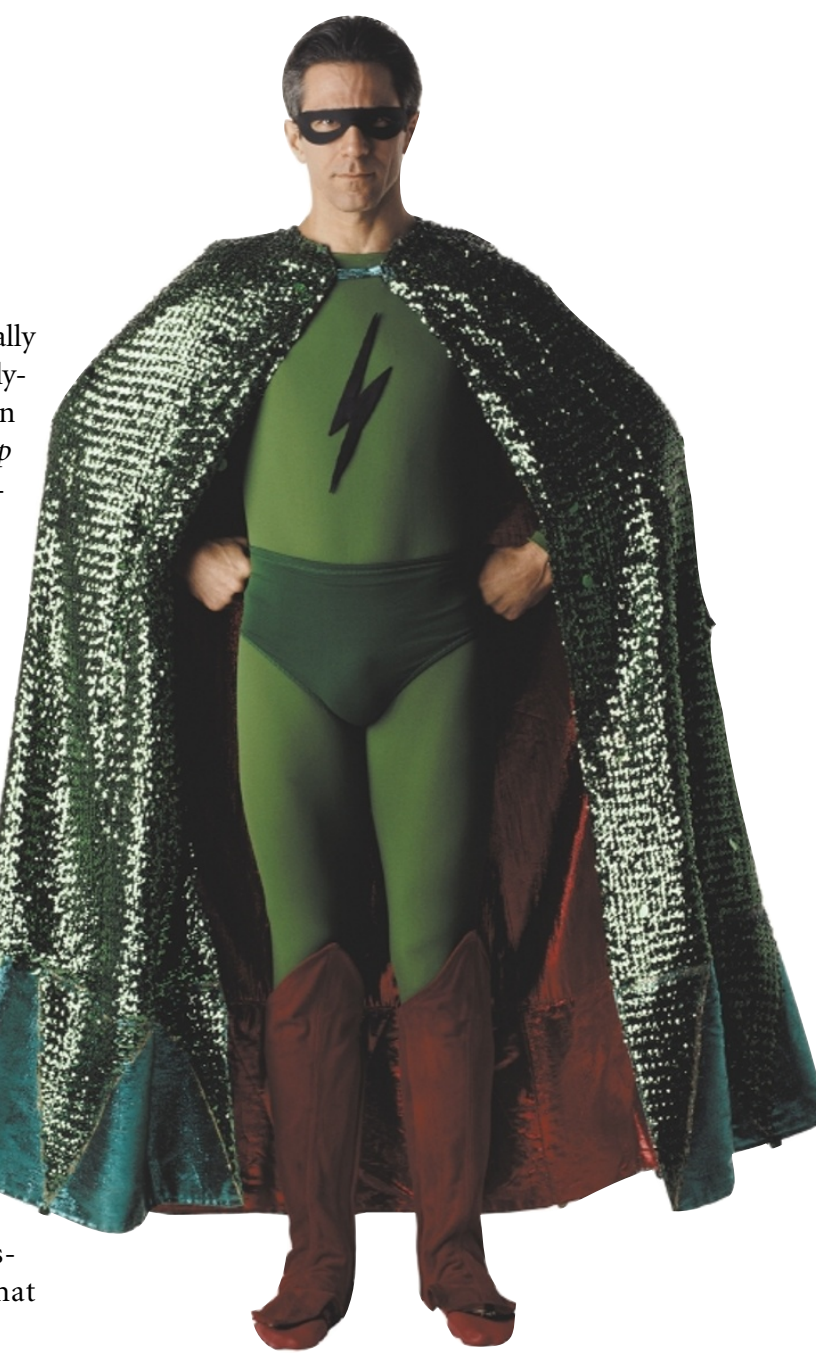


Future research will undoubtedly investigate how specific methylation events are controlled in normal cells and during tumour development. Understanding these mechanisms will have important therapeutic implications: changes in DNA methylation are more readily reversible than mutations or deletions, so a deeper knowledge of how DNA methylation goes awry in cancer might pave the way to restoring the function of tumour-suppressor genes in human cancer.

Barbara Marte,

Editor, Nature Cell Biology

(4) References and links ORIGINAL RESEARCH PAPER Rhee, l. et a DNMT1 and DNTM3b cooperate to silence genes in human cancer cells. Nature 416, 552-556 (2002)

FURTHER READING Ponder, B. A. Cancer genetics. Nature 411, 336-341 (2001) WEB SITE

Bert Vogelstein's lab: http://www.hhmi.org/ research/investigators/vogelstein.html

CMT3 has a chromodomain made the authors wonder whether CMT3 could bind directly to Lys 9 of histone $\mathrm{H} 3$, as does $\mathrm{HP} 1$, which binds to methylated, heterochromatic regions. Their in vitro studies and binding assays of tagged CMT3 and histone $\mathrm{H} 3$ in Escherichia coli gave negative results - instead, CMT3 turned out to bind directly to the Arabidopsis HP1.

On the basis of their findings, the authors propose the following model. HP1 is attracted to Lys9 of histone $\mathrm{H} 3$, once it has been methylated by KYP. HP1 then binds to CMT3, thus bringing it to the site of histone methylation. It is then up to CMT3 to methylate the DNA. Although this model awaits an in vivo validation, it suggests the existence of a eukaryote-wide mechanism for attracting DNA methyltransferases to chromatin, in which HP1 has a central role.

Magdalena Skipper

\section{(1) References and links} ORIGINAL RESEARCH PAPER Jackson, J. P. et al. Control of CpNpG DNA methylation by the KRYPTONITE histone $\mathrm{H} 3$ methyltransferase. Nature 17 March 2002 (DOI 10.1038/nature731) WEB SITE

Steve Jacobsen's lab: http://www.mcdb.ucla. edu/Research/Jacobsen/index.html
SIGNAL TRANSDUCTION

\section{Legless flies for wingless research}

Funny-looking fruitflies have provided the starting point for many interesting lines of research. For example, the Drosophila wingless mutant led to the discovery of the Wingless (or Wnt in vertebrates) signalling pathway, which is highly conserved and is involved in many aspects of development. And now a fly with no legs has helped to unearth two new components in the Wingless pathway. The broader significance of these findings is emphasized by the identification of human counterparts for both components, one of which has been implicated in cancer.

When a cell is exposed to the Wingless signal, reduced protein degradation causes the level of the protein Armadillo ( $\beta$-catenin) to rise in the cytoplasm. Armadillo then translocates to the nucleus where, in combination with the transcription factor Pangolin (TCF), it activates the transcription of key genes. Little is known about this part of the pathway, prompting Kramps et al. to look for new genes that might be involved in transcriptional activation.

They started with a genetic screen for mutants that could suppress an overactive Wingless signalling pathway. As well as finding mutations in genes that encode known components of the pathway, they also found a new locus on chromosome 4. Although welcome, this also created a problem - chromosome 4 doesn't recombine during meiosis, ruling out conventional approaches for genetic mapping. The authors therefore devised a method for generating chromosomal deletions, which they used in a complementation analysis. They defined a critical region of $150 \mathrm{~kb}$, and then sequenced all open reading frames in this interval in the suppressor mutants. One chromosome 4 gene was mutated in several mutants, and they named it legless, after the phenotype of certain legless compound heterozygotes.

Legless protein is located in the nucleus, and epistasis analysis showed that it acts downstream of Armadillo in the Wingless pathway. The authors also identified a second new gene - in a two-hybrid screen - that encodes a protein that binds to Legless. They called this gene pygopusafter a genus of lizards with no legs - because of similarities between the pygopus and legless phenotypes. Further genetic and biochemical experiments led the authors to propose a model for the way these new genes work. The main and perhaps only — function of Legless is to link

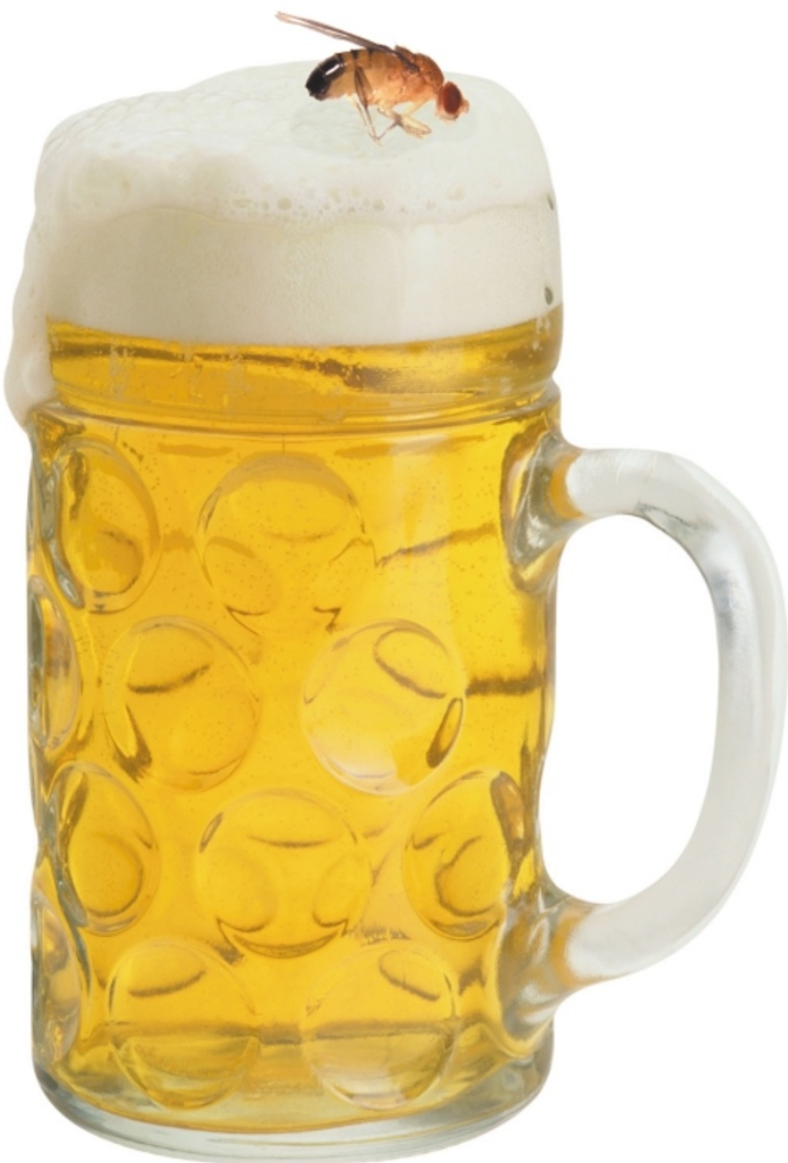

Pygopus to Armadillo in the nucleus. Pygopus then participates in the transcriptional activation of Wingless target genes.

There are human genes related to both legless and pygopus, which can each complement mutations in the fly. Moreover, the functional homologue of legless is $B C L 9$, a gene that has previously been implicated in the development of certain B-cell tumours. The link between Wingless/Wnt signalling and colon cancer is already well established, but these new results suggest that defects in this signal-transduction pathway might underlie other forms of cancer as well, and highlight some interesting new avenues for anticancer therapy. Although there are many details still to figure out, the identification of Legless and Pygopus as two new factors that are specifically required for Wingless/Wnt signalling in the nucleus is a significant step forward.

Mark Patterson

(2) References and links

ORIGINAL RESEARCH PAPER Kramps, T. et al. Wnt/Wingless

signaling requires BCL9/Legless-mediated recruitment of Pygopus

to the nuclear $\beta$ catenin-TCF complex. Cell 15 March 2002 (DOI 10.1016/S0092867402006797)

WEB SITES

Konrad Basler's lab: http://www.unizh.ch/imb/basler/

The Wnt gene homepage:

http://www.stanford.edu/ rnusse/wntwindow.htm 